\title{
High-frequency quasi-periodic oscillations in accreting neutron-star systems
}

\author{
Mariano Méndez \\ Kapteyn Astronomical Institute, University of Groningen, P.O. Box 800, 9700 AV Groningen, \\ The Netherlands \\ email: mariano@astro.rug.nl
}

\begin{abstract}
Kilohertz quasi-periodic oscillations ( $\mathrm{kHz}$ QPOs) are the fastest (almost coherent) variability measured in accreting X-ray binaries with a neutron-star primary. Here I review the rôle of the neutron-star spin in driving the frequencies of the $\mathrm{kHz}$ QPOs.
\end{abstract}

Keywords. stars: neutron, stars: oscillations, X-rays: binaries

\section{Introduction}

Variability is the hallmark of accreting low-mass X-ray binaries (LMXBs), binary systems containing a neutron-star (NS) or black-hole (BH) primary accreting from a normal star via an accretion disc. (In the rest of the paper I will discuss the case of LMXBs with a NS primary, although several, but not all, phenomena mentioned applies also to LMXBs with a BH primary.) Variability in LMXBs covers a broad range of time scales, ranging from several years to milliseconds, and most likely reflects different dynamical aspects of all components in these objects.

The variability over the longest time scales is due to changes in the mass flow onto the primary. It has been recently proposed in Koze \& Charles 2009 that on time scales of years, the driving mechanism is probably activity in the secondary, similar to the activity seen in the Sun. While in this case the amplitude of the variability is generally mild, enhanced X-ray emission may sometimes trigger instabilities in the accretion disc (see, e.g., Cannizzo 1993 for a review), drastically increasing the rate at which mass flows onto the primary, giving rise to a so-called outburst. These outbursts are events in which the X-ray emission of the system increases by large factors within a week or so, remains high for weeks to months, and decays back to low levels within a few days to weeks.

On time scales of years to hours, we see variability due to orbital dynamics of the binary system and the accretion disc. Typical orbital motion of the binary is of the order of a few hours to a few days, while precession of the accretion disc around the primary, or radiation-induced warping of the disc, occurs on time scales of several weeks to months (Whitehurst \& King 1991, Ogilvie \& Dubus 2001). Closer to the primary, on time scales of a few tens of seconds to milliseconds, inhomogeneities in the accretion disc produce quasi-periodic variability in the X-ray light curves of these systems, the so-called quasiperiodic oscillations (QPOs; van der Klis 1989, van der Klis 2006). In NS LMXBs, where there is a surface stopping the accreted matter, variability also reflects instabilities in the burning of the accreted material. In the most extreme cases, unstable burning of the accreted material produces the so-called X-ray bursts, characterized by a sudden flux increase (typical rise times of less than a second) by factors of ten to hundred (Lewin et al. 1993). When the burning finishes, the neutron-star surface cools down and the flux decays back to pre-burst values over a few tens of seconds, and in some extraordinary cases (the so-called super bursts) over few hours to a day (e.g., Cornelisse et al. 2002). 
Also at (or close to) the surface of the NS coherent pulsations, seen both from persistent (non-burst) emission and X-ray bursts, reflect the rotation of the neutron star itself (Wijnands et al. 2003).

Among all these phenomena, the ones with the shortest time scale (or the highest frequency) are the so-called kilohertz QPOs (kHz QPOs) seen in NS LMXBs. (Similar QPOs have been observed in systems containing a $\mathrm{BH}$ primary, at frequencies that are 5 to 10 times lower than in NS systems. The origin of the QPOs in both classes of systems may well be the same.) Although there is still no undisputed model for these oscillations, the time scale involved is tantalizingly close to the crossing time near the surface of the neutron star, therefore understanding these QPOs may bring us closer to probing the highly curved geometry of space time in the vicinity of a neutron star, or to unveil the internal constitution of a neutron star itself.

$\mathrm{KHz}$ QPOs were discovered almost immediately after the launch of the Rossi X-ray Timing Explorer (RXTE; Bradt et al. 1993). The phenomenology of these QPOs is rather complex. Here I will not aim at providing a complete picture of these QPOs (for that see for instance van der Klis 2006), but I will concentrate in a few basic aspects that have guided our attempts to try and understand this phenomenon.

\section{Neutron-star spin and $\mathrm{kHz}$ QPOs}

The $\mathrm{kHz}$ QPOs are relatively narrow peaks that often appear in pairs in the power density spectrum of NS LMXBs, at frequencies $\nu_{1}$ and $\nu_{2}>\nu_{1}$ that change with time. These QPOs are thought to reflect motion of matter at the inner edge of an accretion disc around the neutron star (Miller et al. 1998).

Burst oscillations are short-lived $(\tau \lesssim 10 \mathrm{~s})$, almost coherent pulsations seen at the rise and tail of X-ray bursts in NS LMXBs. The frequency of these oscillations, $\nu_{b}$, increases in the tail of the bursts to an asymptotic value that is consistent with being the same in bursts separated by more than a year time (Strohmayer et al. 1998). This, and the fact that in the accretion-powered millisecond X-ray pulsar (AMP) SAX J1808.4-3658 burst oscillations appear at the same frequency as the pulsations seen during persistent (non-burst) intervals (Wijnands et al. 2003), indicates that the frequency of these burst oscillations is equal to the spin frequency of the NS, $\nu_{s}$.

The idea that the spin of the neutron star is directly involved in the mechanism that produces the $\mathrm{kHz}$ QPOs is deeply entrenched in the community studying this phenomenon. This idea stems from the first detection of $\mathrm{kHz}$ QPOs and burst oscillations in the same source, the LMXB $4 \mathrm{U} 1728-34$, very early on in the $R X T E$ mission. While in different observations the $\mathrm{kHz}$ QPOs appeared at different frequencies, $\nu_{1}$ in the range $\sim 600-800 \mathrm{~Hz}$, and $\nu_{2}$ in the range $\sim 500-1100 \mathrm{~Hz}$, the frequency difference of the QPOs, when both were present simultaneously, was consistent with being constant, $\Delta \nu=\nu_{2}-\nu_{1} \approx 363 \mathrm{~Hz}$, and also consistent with the oscillations seen during bursts in this source at $\nu_{b}=363 \mathrm{~Hz}$ (Strohmayer et al. 1996). This suggested that a beat mechanism with the neutron star spin was responsible for the $\mathrm{kHz}$ QPOs. Further results on other sources appeared to confirm this picture. A detailed model, the sonic-point model(Miller et al. 1998), explained the observed relation between the $\mathrm{kHz}$ QPOs and the neutron star spin in terms of a beat between material orbiting at the inner edge of the disc with the Keplerian frequency at that radius, and the spin of the NS.

As soon as kHz QPOs were discovered in 4U 1636-53 (van der Klis et al. 1996) with a frequency difference of $\Delta \nu=272 \pm 11 \mathrm{~Hz}$, and burst oscillations at a frequency $\nu_{b}=581$ $\mathrm{Hz}$ (Zhang et al. 1996), it became apparent that in this source $\Delta \nu$ was inconsistent with being equal to $\nu_{b}$, but it was close to $\nu_{b} / 2$. This would have been the end of the 


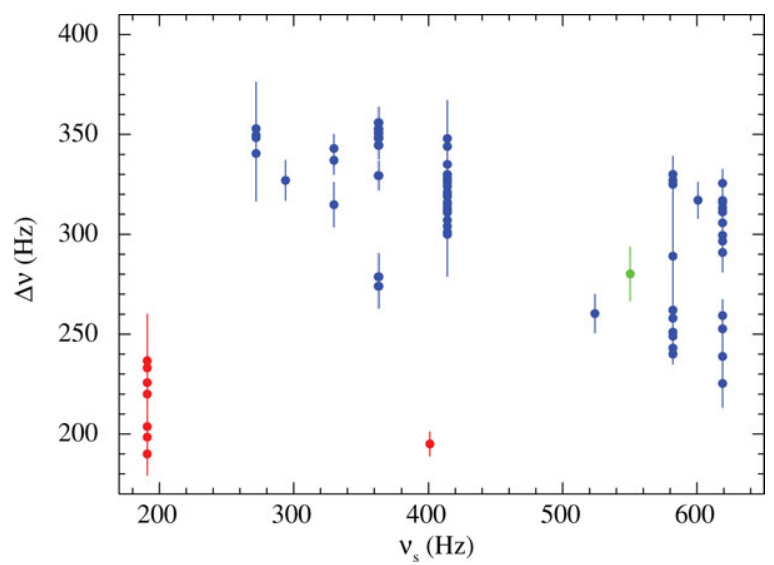

Figure 1. The frequency difference between the two simultaneous $\mathrm{kHz}$ QPOs, $\Delta \nu$ vs. the spin frequency, $\nu_{s}$, for 13 neutron-star LMXBs (see Méndez \& Belloni 2007 for references to the original papers where these data were published). The blue symbols are systems for which the spin was deduced from burst oscillations. The red points are the AMPs SAX J1808.4-3658 and XTE J1807-294. The green point is the transient accreting millisecond X-ray pulsar Aql X-1.

sonic-point model, unless in $4 \mathrm{U} 1636-53$ the $581 \mathrm{~Hz}$ frequency seen during X-ray bursts was the second harmonic of the NS spin frequency, $\nu_{b}=2 \times \nu_{s}$, with $\nu_{s}=290.5 \mathrm{~Hz}$, e.g. if the pulsed radiation came from two antipodal poles on the NS. Although searches for a signal at half the burst oscillations frequency, the putative spin frequency of the neutron star, in the power spectrum of the bursts in $4 \mathrm{U} 1636-53$ yielded no positive result (Strohmayer \& Markwardt (2002)), this option remained viable.

When $\mathrm{kHz}$ QPOs and burst oscillations were discovered in more sources, it became apparent that there was a systematic trend in how $\Delta \nu$ and $\nu_{b}$ were related: For sources for which $\nu_{b} \lesssim 400 \mathrm{~Hz}, \Delta \nu \simeq \nu_{b}$, whereas for sources for which $\nu_{b} \gtrsim 400 \mathrm{~Hz}, \Delta \nu \simeq \nu_{b} / 2$. These two groups of sources were then called "slow" and "fast" rotators, respectively (Muno et al. 2001). But this apparent relation is not without problems. For instance, it is now clear that in several sources $\Delta \nu$ is not constant, but changes in a very definite manner as the QPO frequencies change (e.g., Méndez et al. 1999). More importantly, in the AMP SAX J1808.4-3658, the frequency of burst oscillations is equal to the NS spin frequency (Chakrabarty et al. 2003), while two simultaneous $\mathrm{kHz}$ QPOs with a frequency separation $\Delta \nu \simeq \nu_{s} / 2$ were detected (Wijnands et al. 2003). This result would then also hold for other sources in which $\Delta \nu \simeq \nu_{b} / 2$. The sonic-point model could not explain this.

A testimony of how deep the idea of a direct link between $\mathrm{kHz}$ QPOs and neutron star spin is the fact that despite all this blows, beat-frequency ideas were revived adding small changes to the models in order to account for each new result that seemed to challenge the idea (see, for instance, Lamb \& Miller 2003).

Recently, Méndez \& Belloni (2007) suggested that the data are in fact consistent with a simpler picture in which the frequency separation between $\mathrm{kHz}$ QPOs, $\Delta \nu$, is independent of $\nu_{s}$, with $\langle\Delta \nu\rangle$ being more or less constant across sources. In this interpretation the division between fast and slow rotators is artificial, and is in fact guided by the idea that the NS spin must be directly related to the mechanism that produces the QPOs. In Figure 1 I show $\Delta \nu$ as a function of $\nu_{s}$ for all sources with $\mathrm{kHz}$ QPOs and pulsations or burst oscillations (see Méndez \& Belloni 2007 for the data and references). Except for the two AMPs, SAX J1808.4-3658 and XTE J1807-294, the data are consistent with $\Delta \nu$ being constant, or slightly decreasing with $\nu_{s}$. (The apparent trend of $\Delta \nu$ decreasing with 


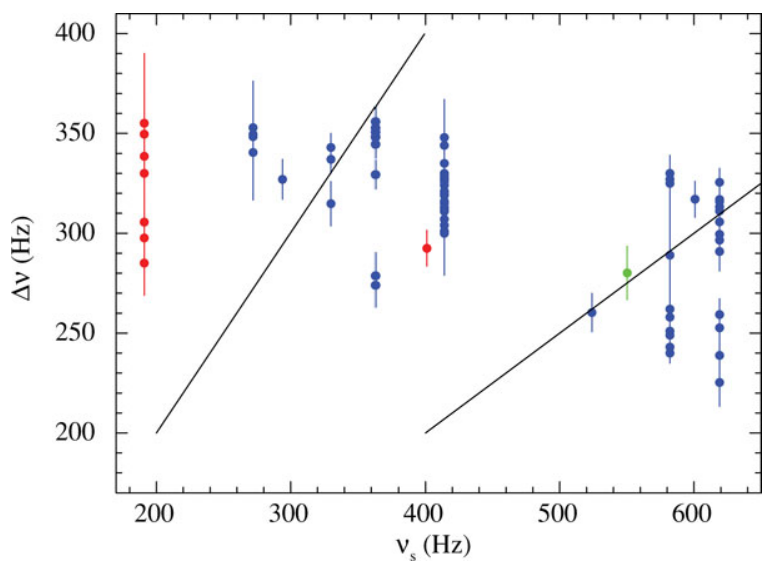

Figure 2. The same as Figure 1, but $\Delta \nu$ of the AMPs SAX J1808.4-3658 and XTE J1807-294 has been multiplied by the factor 1.5 (van Straaten et al. 2005, Linares et al. 2005). The two lines represent the relation $\Delta \nu=\nu_{s}$ for $\nu_{s} \leqslant 400 \mathrm{~Hz}$, and $\Delta \nu=\nu_{s} / 2$ for $\nu_{s} \geqslant 400 \mathrm{~Hz}$, advocated by variants of the beat-frequency model.

$\nu_{s}$ could also be due to the fact that some sources have not been sampled well enough to show a range of $\Delta \nu$ values.)

Several authors have shown that there is a correlation between the frequency of the $\mathrm{kHz}$ QPOs and that of other low-frequency QPOs. More specifically, when plotted vs. the frequency of the upper $\mathrm{kHz} \mathrm{QPO}$, the frequency of the lower $\mathrm{kHz} \mathrm{QPO}$ as well as the frequency of all low-frequency QPOs follow individual correlations that are consistent with being the same in at least eleven sources (see Altamirano et al. 2007 for an overview of these correlations). The AMPs SAX J1808.4-3568 and XTE J1807-214 show similar relations between the frequencies of the low-frequency QPOs and $\nu_{2}$, but the relations are displaced with respect to those of the other sources (van Straaten et al. 2005; Linares et al. 2005). The displacement is best described as a multiplication of $\nu_{2}$ by a factor close to 1.5. While this factor applied to $\nu_{2}$ works for the low-frequency QPOs vs. $\nu_{2}$ correlations, it does not work for the correlation between $\nu_{1}$ and $\nu_{2}$. Interestingly, van Straaten et al. (2005) and Linares et al. (2005) noticed that the $\nu_{1}$ vs. $\nu_{2}$ correlation in the AMPs and in the other sources would be reconciled if they also multiplied $\nu_{1}$ by the same factor that they used to describe the displacement of the $\nu_{2}$ vs. low-frequency QPO correlations. (Notice that $\nu_{1}$ was not used to derive that factor.) The nature of this shift is unclear, but taken at face value, a multiplicative factor applied both to $\nu_{1}$ and $\nu_{2}$ implies that the frequency difference $\Delta \nu=\nu_{2}-\nu_{1}$ must also be multiplied by this factor. In Figure 2 I show the same plot as in Figure 1, but here I have multiplied $\Delta \nu$ of the AMPs SAX J1808.4-3658 and XTE J1807-294 by the factor close to 1.5. For completeness, I also plot the relation predicted by beat-frequency models that advocate the idea that $\Delta \nu=\nu_{s}$ and $\Delta \nu=\nu_{s} / 2$ below and above a certain spin period (in this case I took $400 \mathrm{~Hz}$ as the dividing spin).

\section{Conclusions}

I have briefly described the idea put forward by Méndez \& Belloni (2007), that the frequency difference of the $\mathrm{kHz}$ QPOs, $\Delta \nu$, in neutron-star low-mass X-ray binaries is probably unrelated to the spin frequency, $\nu_{s}$, of the neutron star. This is in contradiction to beat-frequency models that have been proposed to explain the $\mathrm{kHz}$ QPOs. Notice that beat-frequency mechanisms have been originally advanced in the 1980s (see van der Klis 
1989 and references therein) to explain the low-frequency QPOs in these same systems. One cannot rule out completely the hypothesis of a beat that links the $\mathrm{kHz}$ QPOs and the neutron-star spin, but as Méndez \& Belloni 2007 have suggested, the evidence for that link is not as strong as usually assumed. After the paper by Méndez \& Belloni 2007, Strohmayer et al. 2008 discovered oscillations in one burst of the NS LMXB 4U 0614+09 with BAT, on board Swift. The frequency of the oscillations, $\nu_{s}=414.7 \mathrm{~Hz}$, or half that, is significantly different than $\Delta \nu=320 \mathrm{~Hz}$ (e.g., Boutelier et al. 2009). Probably it is time to abandon beat-frequency models and explore other alternatives in more detail (Stella \& Vietri 1999).

\section{Acknowledgements}

The topics described in this short contribution stem from discussions with Tomaso Belloni, with whom we actually wrote a full paper on this. This work was finished under the supervision of my 4-year old son, Manuel, who carefully checked that there were enough uppercase "A's" in the text. The rest of the text is the author's own responsibility.

\section{References}

Altamirano, D., van der Klis, M., Klein-Wolt, M., Méndez, M., van Straaten, S., Jonker, P. G., Lewin, W. H. G., \& Homan, J. 2008, ApJ, 685, 436

Boutelier M., Barret D., \& Miller M. C. 2009, MNRAS, 399, 1901

Bradt H. V., Rothschild R. E., \& Swank J. H. 1993, A\&AS, 97, 355

Cannizzo, J. K. 1993, in: Wheeler, J., (ed.), Accretion Disks in Compact Stellar Systems, World Scientific, Singapore, p. 6.

Chakrabarty D., Morgan E. H., Muno M. P., Galloway D. K., Wijnands R., van der Klis M., \& Markwardt C. B. 2003, Natur, 424, 42

Cornelisse R., et al. 2002, A\&A A, 392, 885

Koze, M. M. \& Charles, P. A. 2009, MNRAS, in press (arXiv:0911.4188)

Lamb F. K. \& Miller M. C. 2003, ApJ, submitted, preprint, arXiv:astro-ph/0308179v1

Lewin, W. H. G., van Paradijs, J., \& Taam, R. 1993, in W. H. G. Lewin, J. van Paradijs, \& E. P. J. van den Heuvel, (eds.), X-ray Binaries, Cambridge Univ. Press, p. 175

Linares, M., van der Klis, M., Altamirano, D., \& Markwardt, C. B. 2005, ApJ, 634, 1250

Méndez, M., van der Klis, M., Wijnands, R., Ford, E. C., \& van Paradijs, J. 1999, ApJ, 511, L49

Méndez, M. \& Belloni, T. 2007, MNRAS, 381, 790

Miller, M. C., Lamb, F. K., \& Psaltis, D. 1998, ApJ, 508, 791

Muno M. P., Chakrabarty D., Galloway D. K., \& Savov P. 2001, ApJ, 553, L157

Ogilvie G. I. \& Dubus G. 2001, MNRAS, 320, 485

Stella, L. \& Vietri, M. 1999, Phys. Rev. Lett, 82, 17

Strohmayer T. E. \& Markwardt C. B. 2002, ApJ, 577, 337

Strohmayer T. E., Markwardt C. B., \& Kuulkers E. 2008, ApJ, 672, L37

Strohmayer T. E., Zhang W., Swank J. H., \& Lapidus I. 1998, ApJ, 503, L147

Strohmayer T. E., Zhang W., Swank J. H., Smale A., Titarchuk L., Day C., \& Lee U. 1996, ApJ, 469, L9

van der Klis, M. 1989, ARA\&A, 27, 517

van der Klis, M. 2006, in: Lewin W., van der Klis M., (eds.), Compact Stellar X-ray Sources, Cambridge Univ. Press, Cambridge, p. 46

van der Klis, M., van Paradijs, J., Lewin, W. H. G., Lamb, F. K., Vaughan B., Kuulkers E., \& Augusteijn, T. 1996, IAUC, 6428, 2

van Straaten, S., van der Klis, M., \& Wijnands, R. 2005, ApJ, 619, 455

Whitehurst R. \& King A. R. 1991, MNRAS, 249, 25

Wijnands, R., van der Klis, M., Homan, J., Chakrabarty, D., Markwardt, C. B., \& Morgan, E. H. 2003, Natur, 424, 44

Zhang, W., Lapidus, I., Swank, J. H., White, N. E., \& Titarchuk, L. 1996, IAUC, 6541, 1 